\title{
Qualidade de vida de idosos com deficiência e prática de atividade física em instituições de longa permanência
}

\author{
Quality of life of elderly with disabilities and physical activity in long-term institutions
}

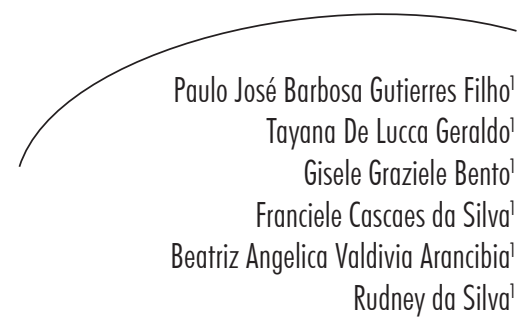

Resumo

Objetivo: Analisar as relações entre qualidade de vida e nível de atividade física de idosos com deficiência que residem em instituições de longa permanência. Método: Este estudo exploratório foi realizado com 55 idosos, por meio de questionários da Organização Mundial da Saúde sobre qualidade de vida (WHOQOL-Bref), específico para idosos (WHOQOL-Old) e sobre atividade física (IPAQ-Long), além de pesquisa em prontuários. Resultados: Constatou-se que a maioria dos idosos estudados foi caracterizada como do sexo feminino, apresentou idade entre 80 e 89 anos; predominância de deficiência visual; maiores escores de qualidade de vida geral nos domínios psicológico e social e menores escores nos domínios físico e ambiental; maiores escores de qualidade de vida relacionada ao envelhecimento nos domínios intimidade e atividade de vida (passadas, presentes e futuras); e menores escores nos domínios morte e medo de morrer e habilidades sensoriais; baixo nível de atividade física e foi considerada sedentária. Verificou-se, ainda, associação significante entre o escore geral de qualidade de vida com comportamentos sedentários e com diversos domínios da qualidade de vida relacionada ao envelhecimento. Conclusão: Os idosos pesquisados com menores tempos sentados, maiores escores nos domínios da autonomia e da intimidade, e menores escores no domínio morte e medo morrer estão associados com maiores escores de qualidade de vida.

Abstract

Objective: To analyze the relationship between quality of life and level of physical activity in elderly with disabilities in long-term institutions. Methods: Exploratory study conducted with 55 elderly, through questionnaires from the World Health Organization about quality of life (WHOQOL-Bref), for elderly (WHOQOL-Old), and on physical activity (IPAQ-Long), besides medical charts. Results: Most elderly were characterized as female, aged 80 to 89 years, showed visual impairment, higher scores for general quality of life in the psychological and social domains and lower scores on the physical and environmental domains; higher scores of quality of life related to aging in the

Palavras-chave: Qualidade de vida. Atividade Motora. Idoso. Pessoas com Deficiência. Instituições de Longa Permanência para Idosos.
Key words: Quality of Life. Motor Activity. Elderly. Disabled Person. Homes for the aged.

\footnotetext{
Laboratório de Atividade Motora Adaptada, Centro de Ciências da Saúde e do Esporte. Universidade do
} Estado de Santa Catarina. Santa Catarina, SC, Brasil. 
intimacy and activity of life (past, present and future) domains and lower scores death and dying and sensory skills domains; low levels of physical activity and were considered sedentary. We also found significant association between the overall score for quality of life with sedentary behavior and various domains of quality of life related to aging. Conclusion: Older respondents with less time sitting down, higher scores in the areas of autonomy and intimacy, and lower scores in the field fear death and dying are associated with higher scores for quality of life.

\section{INTRODUÇÃOO}

O crescimento do envelhecimento populacional vem se mostrando um dos grandes desafios das últimas décadas. Atualmente o Brasil possui aproximadamente 15 milhões de idosos com idades acima dos 65 anos, dos quais $67,7 \%$ possuem algum tipo e grau de deficiência, prevalecendo a visual e a motora. ${ }^{1}$ O envelhecimento é um processo dinâmico, progressivo e irreversível, relacionado a fatores físicos, psíquicos e sociais ${ }^{2}$ que podem provocar condições patológicas ou terminais ${ }^{3}$ associadas às perdas funcionais típicas das deficiências. ${ }^{4}$

Estatísticas oficiais mostram que quase 1,3 milhão de idosos residem em instituições de longa permanência para idosos (ILPIs). ${ }^{1}$ Os fatores que contribuem para esse fenômeno se referem principalmente aos ganhos sociais e econômicos ocorridos nos últimos anos nos sistemas de atendimento e de cuidado ao idoso. ${ }^{3}$ Contudo, as instituições brasileiras ainda apresentam estruturas deficitárias para o cuidado a essa população, que afetam negativamente a saúde e a qualidade de vida do idoso nas ILPIs. ${ }^{5}$

Estudos têm verificado que a qualidade de vida, compreendida como a percepção do indivíduo de sua posição na vida, no contexto da cultura e sistema de valores nos quais ele vive e em relação a seus objetivos, expectativas, padrões e preocupações ${ }^{6}$, piora em idosos que se encontram em ILPIs, a partir de diversos fatores, como isolamento social, redução da rede de apoio, baixa escolarização e diminuição das oportunidades de atividades mentais, físicas e de lazer. ${ }^{6-8}$ Contudo, apesar dos benefícios da atividade física frente ao envelhecimento ${ }^{4,9}$, estudos têm verificado altos índices de sedentarismo em idosos de ILPIs. ${ }^{8,10,11}$
Considerando o exposto, pode-se apontar que se por um lado o aumento da população idosa nas últimas décadas tem provocado o crescimento no número de trabalhos científicos sobre envelhecimento, por outro, pode-se constatar que ainda são poucos os estudos publicados sobre qualidade de vida e atividade física em idosos de ILPIs, ${ }^{12}$ principalmente sobre temáticas relacionadas à deficiência. ${ }^{11}$

Diante desta realidade, o presente estudo teve como objetivo analisar as relações entre qualidade de vida e nível de atividade física de idosos com deficiência residentes em ILPIs. Para tanto, tem-se como hipótese que o baixo nível de atividade física afeta negativamente a qualidade de vida de idosos institucionalizados.

\section{MÉTODO}

Este estudo transversal foi aprovado pelo Comitê de Ética em Pesquisa da Universidade do Estado de Santa Catarina, sob protocolo $n^{\circ}$ $284 / 2009$. Foram tomados cuidados adicionais visando minimizar os riscos envolvidos na pesquisa com populações vulneráveis, quanto a informação detalhada da pesquisa, aplicação dos instrumentos e anuência dos representantes institucionais.

\section{Sujeitos}

O quantitativo amostral foi calculado com o software Biostat 5.0, amplamente utilizado em pesquisas na área das Ciências da Saúde, para testar um coeficiente de correlação de 0,35 , com poder do teste de 0,70 e nível de significância de 0,05, exigindo um mínimo de 50 sujeitos, 
conforme a fórmula: $n=\left(z_{1-a / 2}+z_{1-\beta} / z_{1}\right)^{2}+4$, na qual $z_{r}=0.5 * 1 \mathrm{n}(1+r / 1-\mathrm{r})$. Foram ainda acrescidos $10 \%$ visando compensar possíveis perdas amostrais, totalizando 55 sujeitos (n).

Os sujeitos foram caracterizados como idosos provenientes de ILPIs com deficiências visuais, físicas ou auditivas, identificados na região metropolitana da grande Florianópolis, do Estado de Santa Catarina. Foram incluídos sujeitos que apresentaram idade igual ou superior a 60 anos e condição de deficiência (física e/ou sensorial) não incapacitante à participação no estudo. Excluíram-se os sujeitos que não apresentaram condições psíquicas ou intelectuais, ou que não eram alfabetizados. Os sujeitos apresentaram idade mediana de $81,5 \pm 8$ anos de idade e de ambos os sexos.

\section{Instrumentos}

Foram adotados três instrumentos da Organização Mundial da Saúde para este estudo: (1) Questionário sobre Atividades Físicas, versão longa (IPAQ-Long); (2) Questionário sobre Qualidade de Vida, versão curta (WHOQOLBref); (3) Questionário sobre Qualidade de Vida para Idosos (WHOQOL-Old). Esses instrumentos foram selecionados para uso neste estudo por quatro razões básicas: (1) possuem consistência psicométrica suficiente; (2) possuem ampla aceitação e utilização internacional; (3) possuem confiabilidade e validade para a realidade brasileira; (4) permitem a mensuração quantificada das variáveis-alvos. Além desses instrumentos, foi utilizada uma planilha para coleta de dados demográficos sobre idade, sexo, escolaridade, tipo de deficiência e número de filhos.

O IPAQ-Long foi selecionado ainda para este estudo por fornecer a classificação do nível de atividade física e por ter sido, tanto para idosas ${ }^{13}$ quanto para idosos ${ }^{14}$, adequadamente validado. $\mathrm{O}$ IPAQ-Longé composto de 25 questões que avaliam a atividade física a partir de cinco domínios: trabalho, tarefas domésticas, transporte, lazer/ esporte/recreação e tempo sentado.
O WHOQOL-Bref foi selecionado para este estudo por permitir comparações intergrupos e intra-grupos. É composto de 26 questões que investigam a qualidade de vida nos domínios físico, psicológico, social, ambiental. ${ }^{6}$

WHOQOL-Old é aplicado conjuntamente ao WHOQOL-Bref e é composto por 24 questões direcionadas especialmente para avaliar a qualidade de vida da população idosa nos domínios das habilidades sensoriais, autonomia, atividades de vida (passadas, presentes e futuras), participação social, morte e medo de morrer, e intimidade..$^{15}$

\section{Procedimentos de coleta dos dados}

A seleção dos sujeitos com proveniência na região da grande Florianópolis foi realizada durante os meses de novembro e dezembro de 2009, basicamente por duas razões: (1) grande concentração de unidades de referência no atendimento à saúde; e (2) grande número de instituições de cuidados geriátricos.

Após a seleção da região, foi realizado sorteio de duas das 22 cidades da grande Florianópolis, selecionando-se aleatoriamente o município de Florianópolis e São José. Após o sorteio, foi realizado levantamento junto aos Conselhos Municipais dos municípios selecionados, que identificou 34 instituições de longa permanência (ILPIs) cadastradas.

Foram realizados contatos telefônicos com todas as ILPIs, informando sobre os objetivos da pesquisa e verificando o interesse em participar do estudo. Contudo, 23 ILPIs não concordaram em participar da pesquisa. Os principais motivos identificados para não participação foram: (1) falta de permissão dos proprietários para acesso às ILPIs; (2) falta de permissão dos familiares dos idosos; e (3) falta de condições dos idosos.

Após a obtenção das anuências das 11 ILPIs que concordaram em participar, o estudo foi submetido à avaliação ética. Após a aprovação do Comitê de Ética no mês de dezembro de 2009, foi realizado o levantamento, no mês de janeiro 
de 2010, do quantitativo amostral em cada uma das 11 ILPIs participantes, identificando-se 186 indivíduos. Após os levantamentos, procedeuse ao agendamento dos dias e dos horários das coletas de dados de acordo com a disponibilidade das ILPIs.

A coleta dos dados foi iniciada com pesquisa nos prontuários médicos dos idosos participantes, entre os meses de janeiro e fevereiro de 2010. Este procedimento permitiu identificar as variáveis demográficas (idade, sexo, escolaridade, número de filhos) e clínicas (condição física e/ou sensorial, existência de doenças).

Após a pesquisa nos prontuários, foram identificados os idosos que apresentavam deficiências físicas e/ou sensoriais, aos quais foram apresentados todos os procedimentos do estudo e foi solicitada a participação na pesquisa. Os dados foram coletados somente após a assinatura do Termo de Consentimento Livre e Esclarecido pelos participantes do estudo.

A aplicação dos instrumentos foi realizada entre os meses de março e junho de 2010, em locais reservados que foram disponibilizados pela ILPIs. Os idosos que não possuíam condições motoras e/ou visuais para preenchimento das questões eram auxiliados pelos pesquisadores, que realizavam o preenchimento e a imediata checagem junto aos idosos.

\section{Tratamento dos dados}

Os valores de referência do IPAQ-Long foram calculados segundo diretrizes do Instituto Karolinska da Suécia, ${ }^{16}$ que permitem classificar o nível de atividade física de acordo com o equivalente metabólico (MET), como baixo (até 600 METs ), moderado (entre 601 e 1.500 METs ) e alto (entre 1.501 e 3.000 METs); e a condição como sedentário (até $600 \mathrm{METs}$ ), irregularmente ativo (tipo A entre 601 e 1.500 METs; tipo B entre 1.501 e $3.000 \mathrm{METs}$ ) e muito ativo (mais de 3.000 METs). Os valores de referência do
WHOQOL-Old foram calculados conforme critérios da Organização Mundial da Saúde,${ }^{15} \mathrm{em}$ escore variável entre 0 e 100 pontos.

A tabulação dos dados foi efetuada por meio do programa Microsoft Excel $^{\circledR}(2010)$ e a estatística mediante o programa Statistical Packeage Social Science (SPSS) versão 20.0. Os dados foram tratados por meio da estatística descritiva (média, desviopadrão para dados quantitativos; frequência simples e percentual para dados qualitativos). Adotou-se um intervalo de confiança de 95\% (IC95\%). A normalidade dos dados foi realizada com o teste Kolmogorov-Sminorv. A confiabilidade dos resultados dos instrumentos aplicados foi calculada por meio do coeficiente Cronbach, adotando um valor de alfa igual ou superior a 0,70, tanto para a totalidade dos itens avaliados, quanto para os itens estandardizados.

A comparação da qualidade de vida e o nível de atividade física entre os idosos segundo o sexo foi testada por meio do teste U de Mann-Whitney. Já a associação entre a classificação do nível de atividade física e o sexo foi avaliada por meio do teste qui-quadrado. A relação entre a variável desfecho e as variáveis preditoras foi analisada por regressão linear.

Para regressão linear, considerou-se o escore total obtido do WHOQQL-Bref como variável dependente e os fatores demográficos (idade, sexo, tipo de deficiência), de percepção dos domínios de bem-estar no envelhecimento (habilidades sensoriais, autonomia, atividades passadas, presentes e futuras, participação social, morte e medo de morrer, intimidade) e dos domínios da atividade física (de transporte, de lazer, de caminhada, de atividades moderadas, de atividades vigorosas, tempo em comportamentos sedentários) como variáveis independentes.

As variáveis independentes foram analisadas individualmente em relação à variável dependente, segundo um modelo de regressão linear simples com ponto de corte para entrada no modelo múltiplo, assumindo-se valor de p menor ou igual 
a 0,20. A imputação das variáveis no modelo múltiplo foi realizada pelo método stepwise, sendo consideradas significantes quando o $\mathrm{p}$ fosse menor que 0,05 e/ou quando modificassem em mais de $10 \%$ o valor do coeficiente angular $(\beta)$ de qualquer variável do modelo.

O modelo múltiplo pode ser descrito segundo a equação $Y=\beta_{0}+\beta_{1} X+\beta_{2} X+\ldots+\beta_{\mathrm{n}} X$, na qual $Y$ é o valor predito da variável dependente, $\beta_{0}$ é o intercepto do eixo $Y$ (valor da variável $Y$, quando $X=0$ ), e $\beta_{1}$ é a inclinação da reta (coeficiente angular: valor do incremento de $Y$ para cada unidade de $X$, quando todas as outras variáveis independentes permanecem constantes).

\section{RESULTADOS}

As análises dos resultados dos coeficientes de Cronbach dos questionários WHOQOL-Bref $(a=0,737)$, WHOQOL-Old $(a=0,836)$ e IPAQLong $(a=0,767)$ apontam valores adequados de confiabilidade para as respostas destes instrumentos, segundo o critério adotado ( $\mathrm{p} \geq 0,70)$.

Os valores frequenciais das características demográficas apontam que a maioria dos idosos era do sexo feminino, na faixa etária entre 80 e 89 anos, cursou o ensino fundamental, possuía entre 0 e 2 filhos e apresentava deficiência visual (tabela 1).

Tabela 1. Características demográficas de idosos com deficiência residentes em ILPIs. Florianópolis e São José-SC, 2010.

\begin{tabular}{|c|c|c|}
\hline Variávies & $\mathrm{n}$ & $\%$ \\
\hline \multicolumn{3}{|l|}{ Sexo } \\
\hline Masculino & 12 & 21,8 \\
\hline Feminino & 43 & 78,2 \\
\hline \multicolumn{3}{|l|}{ Idade (anos) } \\
\hline $60-69$ & 6 & 10,9 \\
\hline 70-79 & 18 & 32,7 \\
\hline $80-89$ & 25 & 45,5 \\
\hline 90 ou mais & 6 & 10,9 \\
\hline \multicolumn{3}{|l|}{ Escolaridade } \\
\hline Não escolarizado & 13 & 23,6 \\
\hline Ensino fundamental & 31 & 56,4 \\
\hline Ensino médio & 3 & 5,5 \\
\hline Ensino superior & 8 & 14,5 \\
\hline \multicolumn{3}{|l|}{ Número de filhos } \\
\hline Entre $0-2$ & 29 & 52,7 \\
\hline Entre 3-5 & 18 & 32,8 \\
\hline Entre 6-8 & 2 & 3,6 \\
\hline 9 ou mais & 6 & 10,9 \\
\hline \multicolumn{3}{|l|}{ Tipos de deficiência } \\
\hline Física & 1 & 1,8 \\
\hline Visual & 20 & 36,4 \\
\hline Auditiva & 2 & 3,6 \\
\hline Física e visual & 9 & 16,4 \\
\hline Visual e auditiva & 18 & 32,7 \\
\hline Física, visual e auditiva & 5 & 9,1 \\
\hline
\end{tabular}


O valor médio da qualidade de vida calculado a partir do WHOQOL-Bref aponta escore geral de $57,5 \pm 6,1$ pontos, que pode ser considerado regular. Os menores escores de qualidade de vida foram identificados nos domínios físico e ambiental; e os maiores escores, nos domínios psicológico e social (tabela 2).

O valor médio da qualidade de vida específica de idosos, calculado a partir do WHOQOLOld, obteve escore geral de 49,9 $\pm 6,3$ pontos. Os menores escores de qualidade de vida específica de idosos foram identificados nos domínios "morte e medo de morrer" e "habilidades sensoriais", e os maiores escores nos domínios "intimidade" e "atividade de vida" (passadas, presentes e futuras), segundo a tabela 2.

O valor médio geral do total das atividades físicas, calculado a partir do IPAQ-Long, foi de

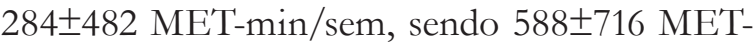
min/sem para os homens e 199 \pm 363 MET-min/ sem para mulheres - ou seja, o nível de atividade física pode ser considerado baixo, não atingindo o mínimo recomendado para manutenção da saúde (tabela 2).

Tabela 2. Valores médios e de desvio-padrão dos domínios da atividade física e da qualidade de vida de idosos com deficiência residentes em ILPIs, por sexo. Florianópolis e São José-SC, 2010.

\begin{tabular}{|c|c|c|c|c|c|c|}
\hline & \multicolumn{2}{|c|}{ Homens } & \multicolumn{2}{|c|}{ Mulheres } & \multicolumn{2}{|c|}{ Geral } \\
\hline & média & $\mathrm{dp}$ & média & $\mathrm{dp}$ & média & $\mathrm{dp}$ \\
\hline \multicolumn{7}{|l|}{ Domínios do IPAQ-Long* } \\
\hline Trabalho & - & - & - & - & - & - \\
\hline Transporte & 308 & 346 & 166 & 282 & 197 & 299 \\
\hline Tarefas domésticas & - & - & - & - & - & - \\
\hline Lazer & 280 & 547 & 33 & 152 & 86 & 299 \\
\hline Leve & 308 & 346 & 166 & 282 & 197 & 299 \\
\hline Moderada & - & - & - & - & - & - \\
\hline Vigorosa & - & - & - & - & - & - \\
\hline Tempo sentado (min/sem) $\dagger$ & 1.738 & 55,2 & 1.769 & 35,1 & 1.760 & 43,1 \\
\hline \multicolumn{7}{|l|}{ Domínios do WHOQOL-Brefキ } \\
\hline Físico & 50,4 & 10,2 & 4767 & 10,7 & 48,3 & 10,5 \\
\hline Psicológico & 63,2 & 6,8 & 60,9 & 8,1 & 61,4 & 7,8 \\
\hline Social & 65,3 & 9,9 & 63,2 & 7 & 63,4 & 8,2 \\
\hline Meio ambiente & 55,2 & 7 & 57 & 6,1 & 56,6 & 6,2 \\
\hline \multicolumn{7}{|l|}{ Domínios do WHOQOL-Oldキ } \\
\hline Habilidades sensoriais & 49 & 14,6 & 45,2 & 13,8 & 46 & 13,9 \\
\hline Autonomia & 55,21 & 9,6 & 48,7 & 9,7 & 50,1 & 9,9 \\
\hline Atividades de vida & 57,3 & 11,9 & 53,8 & 10,9 & 54,5 & 11,1 \\
\hline Participação social & 49,5 & 13,4 & 50,9 & 14 & 50,6 & 13,8 \\
\hline Morte e medo de morrer & 35,4 & 24,8 & 29,2 & 19,4 & 30,6 & 20,6 \\
\hline Intimidade & 67 & 9,3 & 68 & 12,6 & 67,8 & 1,9 \\
\hline
\end{tabular}

*, em metros; $\dagger$, min/sem: minutos por semana; $\neq$, em pontos. 
A comparação da qualidade de vida e o nível de atividade física testada por meio do teste $U$ de Mann-Whitney não revelou diferença significativa entre os idosos segundo o sexo, ou seja, todos os sujeitos se encontravam nas mesmas condições.

Os valores frequenciais referentes ao nível de atividade física apontam que os sujeitos foram classificados como sedentários, principalmente entre as mulheres, já que metade dos homens foi classificada como relativamente ativa (tipo A e tipo B), segundo a tabela 3. A aplicação do teste qui-quadrado apontou associação significante entre sexo e nível de atividade física ( $\mathrm{p}=0,046)$.

A análise de correlação de Spearman evidenciou correlações entre atividades físicas do tipo caminhada, atividades físicas de lazer, atividades físicas de transporte, total das atividades físicas, tempo sentado, domínio da autonomia, domínio da intimidade, domínio da morte e medo morrer, as quais foram inseridas no modelo de regressão linear múltipla. A tabela 4 mostra os valores obtidos por meio da análise de regressão linear múltipla.

Tabela 3. Valores frequenciais e percentuais da classificação do nível de atividade física de idosos com deficiência residentes em ILPIs por sexo. Florianópolis e São José-SC, 2010.

\begin{tabular}{lcccccc}
\hline & \multicolumn{2}{c}{ Homens } & \multicolumn{2}{c}{ Mulheres } & \multicolumn{2}{c}{ Geral } \\
\cline { 2 - 7 } \multicolumn{1}{c}{ Nível de atividade física } & $\mathrm{n}$ & $\%$ & $\mathrm{n}$ & $\%$ & $\mathrm{n}$ & $\%$ \\
\hline Sedentário & 6 & 50 & 34 & 79,1 & 40 & 72,7 \\
Relativamente ativo tipo A & 3 & 25 & 8 & 18,6 & 11 & 20 \\
Relativamente ativo tipo B & 3 & 25 & 1 & 2,3 & 4 & 7,3 \\
\hline
\end{tabular}

Tabela 4. Parâmetros da regressão linear múltipla das variáveis que permaneceram no modelo múltiplo e respectivos valores de B, t e p de cada variável. Florianópolis e São José-SC, 2010.

\begin{tabular}{lccc}
\hline \multicolumn{1}{c}{ Variável } & B* & t† & p \\
\hline Tempo sentado (por aumento de minutos) & $-0,051$ & $-2,796$ & 0,009 \\
Domínio autonomia (por aumento de escore) & 0,278 & 3,225 & 0,003 \\
Domínio intimidade (por aumento de escore) & 0,142 & 2,215 & 0,035 \\
Domínio morte e medo de morrer (por aumento de escore) & $-0,069$ & $-2,061$ & 0,049 \\
\hline
\end{tabular}

*, valor do coeficiente de regressão; $\dagger$, valor da estatística; $\neq$, valor da probabilidade de aceitação de erro tipo I.

As análises dos coeficientes de determinação $\left(\mathrm{R}^{2}\right)$ indicam que $58,4 \%$ da variação do escore geral da qualidade de vida podem ser atribuídos à variação do tempo total sentado, do domínio da autonomia, do domínio da intimidade e do domínio morte e morrer. Juntas, as variáveis explicativas preveem a variável dependente $(F(1,54)=11,893 ; \mathrm{p}<0,001)$.
As análises dos coeficientes de regressão permitem apontar que, quando o tempo sentado aumenta uma unidade de equivalente metabólico (MET), o escore total da qualidade de vida diminui em - 0,051 pontos, com $95 \%$ de confiança de que o coeficiente da população está entre $-0,089$ e - 0,014 . Assim como o aumento de uma unidade de pontos no domínio "autonomia", o 
escore total da qualidade de vida aumenta em 0,278, com $95 \%$ de confiança de que o coeficiente da população está entre 0,101 e 0,455.

Ainda é possível observar que, quando o domínio da intimidade aumenta um ponto, o escore total da qualidade de vida aumenta em 0,142, com 95\% de confiança de que o coeficiente da população está entre 0,011 e 0,274. Do mesmo modo, quando o domínio "morte e medo de morrer" aumenta um ponto, o escore total da qualidade de vida diminui em -0,069, com $95 \%$ de confiança de que o coeficiente da população está entre -0,137 e 0,000 (tabela 4).

\section{DISCUSSÃO}

Os resultados apontam predominância do sexo feminino, com idade igual ou superior a 80 anos e baixo nível de escolaridade. São corroborados por diversas pesquisas realizadas com idosos provenientes de ILPIs, ${ }^{5,7,12,17-22}$ pois a mulher apresenta uma expectativa de vida superior à do homem por diversos motivos relacionados à diferença de oportunidades econômicas, sociais, educacionais e culturais típicas da época em que viveram. ${ }^{23,24}$ Além destes fatores, Guralnik, Balfour \& Volpato ${ }^{24}$ destacam que as mulheres dispensam mais atenção à saúde ao longo da vida e buscam acompanhamento médico quando necessário.

A prevalência de deficiência visual é corroborada por outros estudos, ${ }^{25,26}$ tendo sido descrita na literatura especializada ${ }^{3,4}$ e observada em estudos oriundos da mesma região deste estudo $^{25}$ e do Brasil, ${ }^{26}$ que apontam que o avanço da idade aumenta a possibilidade de desenvolvimento de alguns tipos de deficiência, principalmente a visual e auditiva. Em estudo realizado por Benedetti, Petroski \& Gonçalves ${ }^{25}$ com idosos de Florianópolis-SC, foi verificado que quase um terço dos investigados considerava sua visão como ruim ou péssima. Já estudo de Coelho Filho \& Ramos $^{26}$ apontou que pouco mais de um quarto dos idosos pesquisados possuía deficiência auditiva.
Os escores gerais de qualidade de vida específica de idosos, avaliada pelo WHOQOLOld, abaixo dos 50 pontos, considerados nesta pesquisa como baixos, também são confirmados por diversos estudos ${ }^{7,19} \mathrm{com}$ idosos de ILPIs que constataram que, no geral, a percepção de qualidade de vida desta população está próxima da metade da pontuação.

Os escores gerais de qualidade de vida avaliada pelo WHOQOL-Bref considerados como moderadamente baixos, são amparados por diversos estudos ${ }^{4,719}$ com idosos de ILPIs que constataram que a percepção de qualidade de vida nesta faixa etária está próxima da metade da pontuação. Os escores mais altos no domínio social também são apontados por estudos ${ }^{7,27,28}$ que indicam que o convívio em ILPIs favorece as relações e o apoio dos moradores e cuidadores que formam uma nova rede social de apoio, apesar das ausências parentais.

Deve-se destacar, ainda, que os domínios "autonomia" e "intimidade" tiveram alguns dos mais altos escores da qualidade de vida avaliada com o WHOQOL-Brief. Estes resultados divergem do estudo de Dalsenter \& Matos, ${ }^{29}$ no qual 45,8\% dos idosos institucionalizados da cidade de Blumenau-SC referem-se insatisfeitos no domínio autonomia - ou seja, na liberdade de gerenciar sua própria vida. Do mesmo modo, os maiores escores do domínio intimidade podem indicar que os idosos investigados estão tão satisfeitos ou estão mantendo relações de intimidade suficientes que lhe permitem perceber positivamente a qualidade de vida como um todo. Já estes resultados são corroborados pelo estudo de Bajotto \& Goldim, ${ }^{30}$ que destaca o companheirismo, o amor e a visão madura da vida, apesar do conformismo com os relacionamentos pessoais e íntimos, como fatores relevantes da qualidade de vida de idosos. Nesse sentido, as análises preditivas permitem explicar o aumento da qualidade de vida a partir do aumento do domínio autonomia e do domínio intimidade.

Já os escores mais baixos no domínio físico são corroborados por alguns estudos ${ }^{7,27,31}$ que 
apontam que as limitações físicas afetam a autonomia e, consequentemente, a percepção sobre os fatores físicos acentuam-se com a ocorrência de deficiências ${ }^{32}$ e provocam graves impedimentos, principalmente na mobilidade dos idosos. ${ }^{3,4}$ Esse mecanismo também foi verificado na avaliação da mobilidade dos idosos, já que o nível de atividade física foi classificado como baixo, o que implica que este idosos não atingem o mínimo recomendado para manutenção da saúde de acordo com as recomendações atuais de, pelo menos, 30 minutos diários de atividade física com intensidade moderada ou vigorosa. ${ }^{33,34}$

Contudo, as análises preditivas apontaram que a diminuição do escore geral da qualidade de vida pode ser atribuída ao aumento do tempo total sentado, o que também já foi observada em estudos com idosas brasileiras ${ }^{34}$ e estrangeiras. ${ }^{31}$ Isso pode ser compreendido pelos comportamentos sedentários adotados pelos idosos, que permanecem por longo tempo assistindo televisão, locomovendo-se utilizando automóveis, ouvindo rádio, costurando, lendo e recebendo visitas. Estas considerações são sustentadas, ainda, pela literatura especializada, ${ }^{33,35}$ que aponta que apesar dos benefícios da atividade física para a qualidade de vida de idosos, a inatividade física ainda é um dos maiores desafios de saúde pública nesta população.

Os resultados apontam, ainda, que o domínio "morte e morrer" apresenta menor escore de qualidade de vida avaliada pelo WHOQOL-Brief. Deste modo, pode-se explicar a diminuição da qualidade de vida a partir do domínio de morte e morrer. Contudo, a literatura especializada ${ }^{3}$ sugere que mesmo idosos com idades avançadas encaram a morte de forma mais natural, o que pode ser corroborado pelo Torres et al. ${ }^{21}$ que destacam a pouca preocupação e a coragem dos idosos quando o assunto é a morte.

Deve-se informar que as limitações deste estudo estão associadas às pesquisas envolvidas com populações vulneráveis, neste caso: (a) perda amostral pelas restrições dos sujeitos de pesquisa; (b) perda amostral pelas garantias de autonomia do sujeito sem intenções de participar da pesquisa; (c) perda amostral pelas restrições das instituições; aos instrumentos adotados, neste caso: d) necessidade da memória e da lembrança para o preenchimento do IPAQ; e) restrição da percepção subjetiva da qualidade de vida por respostas fechadas do tipo Likert do WHOQOL e do WHOQOL-Brief; e ainda aos procedimentos adotados, neste caso: f) a obtenção das informações somente pelo relato do participante, o que pode ter gerado, mesmo que em pequeno grau, viés de informação. Apesar das limitações, o presente estudo forneceu informações relevantes sobre as relações entre qualidade de vida e nível de atividade física de idosos com deficiência que residem em instituições de longa permanência, sobretudo que, apesar de estar consolidado na literatura especializada o papel positivo do exercício físico, conforme a hipótese de trabalho adotada, os comportamentos sedentários também influenciam a qualidade de vida desses sujeitos.

Finalmente, destaca-se que os resultados deste estudo não devem ser generalizados a todos os idosos residentes de instituições de longa permanência, pois foram avaliados idosos de 11 ILPIs de dois municípios de Santa Catarina. Além disso, a natureza do estudo só permitiu estabelecer associações entre as variáveis, e não relações de causa-efeito, o que sugere a necessidade de mais estudos sobre a temática e com diferentes delineamentos metodológicos.

\section{CONCLUSÃO}

Pode-se concluir que os idosos com deficiência residentes nas instituições de longa permanência pesquisadas com menores tempos sentados, maiores escores nos domínios "autonomia" e "intimidade" e menores escores no domínio morte e medo morrer estão associados com maiores escores de qualidade de vida, independentemente de cada uma das variáveis independentes finais. 
Sendo assim, sugere-se a aplicação dos consensos atuais sobre a promoção da prática de atividades físicas aos idosos residentes em ILPIs, pois um estilo de vida ativo é uma estratégia eficaz de promoção da saúde, funcionalidade, autonomia e melhor qualidade de vida dos idosos.

\section{REFERÊNCIAS}

1. Instituto Brasileiro de Geografia e Estatística. Séries Estatísticas e Séries Históricas. Brasília: IBGE; 2013.

2. Brito FC, Litvoc CJ. Envelhecimento: prevenção e promoção de saúde. São Paulo: Atheneu; 2004.

3. Birren JE, Schaie KW. Handbook of the psychology of aging. San Diego: Academic Press; 1996.

4. Freitas EV, Py L, Neri AL, Cançado FAX, Gorzoni ML, Rocha SM. Tratado de geriatria e gerontologia. Rio de Janeiro: Guanabara Koogan; 2002.

5. Mincato PC, Freitas CD. Qualidade de vida dos idosos residentes em instituições asilares da cidade de Caxias do Sul - RS. RBCEH 2007;4(1):127-38.

6. Fleck MPA, Louzada S, Xavier M, Chachamovich E, Vieira G, Santos L, et al. Aplicação da versão em português do instrumento abreviado de avaliação da qualidade de vida "WHOQOL-bref". Rev Saúde Pública 2000;34(2):178-83.

7. Vitorino LM, Paskulin LMG, Viana LAC. Qualidade de vida de idosos em instituições de longa permanência. Rev Latinoam Enferm 2012;20(6):1186-95.

8. Carneiro RS, Falcone E, Clark C, Del Prette Z, Del Prette A. Qualidade de vida, apoio social e depressão em idosos: relação com habilidades sociais. Psicol Reflex Crit 2007;20(2):229-37.

9. Shephard RJ. Envelhecimento, atividade física e saúde. São Paulo: Phorte; 2003.

10. Binotto MA, Borgatto AF, Farias SF. Nível de atividade física: Questionário Internacional de Atividades Físicas e tempo de prática em mulheres idosas. Rev Bras Geriatr Gerontol 2010;13(3):425-34.

11. Carneiro R, Falcone E. Um estudo das capacidades de deficiências em habilidades sociais na terceira idade. Psicol Estud 2004;9(1):119-26.

12. Freitas MAV, Scheicher ME. Qualidade de vida de idosos institucionalizados Rev Bras Geriatr Gerontol 2010;13(3):395-401.

13. Benedetti TB, Mazo GZ, Barros MVG. Aplicação do Questionário Internacional de Atividades Físicas para avaliação do nível de atividades físicas de mulheres idosas: validade concorrente e reprodutibilidade testereteste. Rev Bras Ciênc Mov 2004;12(1):25-34.
14. Benedetti TRB, Antunes PC, Rodriguez-Añez CR, Mazo GZ, Petroski EL. Reprodutibilidade e validade do questionário internacional de atividade física (IPAQ) em homens idosos. Rev Bras Med Esporte 2007;13(1):11-6.

15. Power M, Quinn k, Schmidt S; WHOQOL-old Group. Development of the WHOQOL-old module. Qual Life Res 2005;14(10):2197-214.

16. International Physical Activity Questionnaire. Guidelines for data processing and analysis of the International Physical Activity Questionnaire. Stockholm: IPAQ; 2005.

17. Barcelos-Ferreira R, Nakano EY, Steffens DC, Bottino CM. Quality of life and physical activity associated to lower prevalence of depression in community-dwelling elderly subjects from São Paulo. J Affect Disord 2013;150(2):616-22.

18. Carli L, Kolankiewicz ACB, Loro MM, Rosanelli CLSP, Stumm EFM, Pettenon MK. Ambiente asilar: única opção de moradia. Rev Contexto \& Saúde 2011;10(20):647-52

19. Cader SA, Guimarães AC, Rocha CAQC, Vale RGS, Pernanbuco CS, Dantas EHM. Perfil da qualidade de vida e da autonomia funcional de idosos asilados em uma instituição filantrópica no município do Rio de Janeiro. Fitness \& Perform J 2006;5(4):256-61.

20. Danilow MZ, Moreira ACS, Vilela CG, Barra BB, Garbi MRC, Oliveira MPF. Perfil epidemiológico, sociodemográfico e psicossocial de idosos institucionalizados do Distrito Federal. Comun Ciênc saúde 2007;18(1):9-16.

21. Torres GV, Reis LA, Reis LA, Fernandes MH. Qualidade de vida e fatores associados em idosos dependentes em uma cidade do interior do Nordeste. J Bras Psiquiatr 2009;58(1):39-44.

22. Lenardt MH, Michel T, Tallmann AE. A condição de saúde de idosas residentes em instituição de longa permanência. Cogitare Enferm 2009;14(2):227-36.

23. Camargos MCS, Perpétuo IHO, Machado CJ. Expectativa de vida com incapacidade funcional em idosos em São Paulo, Brasil. Rev Panam Salud Pública 2005;17(5/6):379-86. 
24. Guralnik JM, Balfour JL, Volpato S. The ratio of older women to men: historical perspectives and cross-national comparisons. Aging (Milano) 2000;12(2):65-76.

25. Benedetti TB, Petroski EL, Gonçalves LT. Condições de saúde nos idosos de Florianópolis. ACM Arq Catarin Med 2006;35(1):44-51.

26. Coelho Filho JM, Ramos LR. Epidemiologia do envelhecimento do Brasil: resultados de inquérito domiciliar. Rev Saúde Pública 1999;33(5):445-53.

27. Araújo CLO, Onofre AA, Silva ET, Pena MVD. Qualidade de vida de idosos institucionalizados. Rev Kairós 2010:35-44.(Caderno Temático, vol. 8).

28. Oliveira ERA, Gomes MJ, Paiva KM. Institucionalização e qualidade de vida de idosos da região metropolitana de Vitória - ES. Esc Anna Nery 2011;15(3):618-23.

29. Dalsenter CA, Matos FM. Percepção da qualidade de vida em idosos institucionalizados da cidade de Blumenau (SC). Dynamis 2009;15(2):32-7.

30. Bajotto AP, Goldim JR. Avaliação da qualidade de vida e tomada de decisão em idosos participantes de grupos socioterápicos da cidade de Arroio do Meio, RS, Brasil. Rev Bras Geriatr Gerontol 2011;14(4):753-61.

31. Gierach GL, Chang SC, Brinton LA, Lacey Junior JV, Hollenbeck AR, Schatzkin A, et al. Physical activity, sedentary behavior, and endometrial cancer risk in the NHI-AARP diet and health study. Int J Cancer 2009;124(9):2139-47.

32. Silva RB, Costa-Paiva L, Pinto Neto AM, Braga AA, Morais SS. Atividade física habitual e risco cardiovascular na pós-menopausa. Rev Assoc Med Bras 2006;52(4):242-6.

33. Vogel T, Lang PO, Schmitt G, Kaltenbach G, Geny B. Health benefits of physical activity in older patients: a review. Cahiers Année Gérontol 2013;5(3):257-67.

34. Mazo GZ, Mota J, Gonçalves LHT, Matos GM. Nível de atividade física, condições de saúde e características sócio-demográficas de mulheres idosas brasileiras. Rev Port Cien Desp 2005;5(2):202-12.

35. Vogel T, Brechat PH, Leprêtre PM, Kaltenbach G, Berthel M, Lonsdorfer J. Health benefits of physical activity in older patients: A review. Int J Clin Pract 2009;63(2):303-20. 